\title{
NIR integral field spectroscopy of high mass young stellar objects
}

\author{
K. Murakawa ${ }^{1}$, S. L. Lumsden ${ }^{1}$, R. D. Oudmaijer ${ }^{1}$, \\ B. Davies ${ }^{2}$ and M. G. Hoare ${ }^{1}$ \\ ${ }^{1}$ School of Physics and Astronomy, University of Leeds, Leeds LS2 9JT, United Kingdom \\ email: k.murakawa@leeds.ac.uk \\ ${ }^{2}$ Institute of Astronomy, University of Cambridge, Cambridge CB3 0H, United Kingdom
}

\begin{abstract}
We present K-band Integral Field Spectroscopy of six high mass young stellar objects (IRAS 18151-1208, AFGL 2136, S106 IRS4, V645 Cyg, IRAS 19065+0526, and G082.5682+ 00.4040) obtained using the adaptive optics assisted NIFS instrument mounted on the Gemini North telescope. The targets are chosen from the Red MSX Source survey led by University of Leeds. The data show the spectral features of $\mathrm{Br} \gamma, \mathrm{H}_{2}$, and gas phase $\mathrm{CO}$ emissions and absorptions with a spectral resolution of $R \approx 5500$, which allow a three-dimensional spectroastrometric analysis of the line emissions. We discuss the results of the ionized jets and winds, and rotating $\mathrm{CO}$ torus.
\end{abstract}

Keywords. astrometry — stars: winds, outflows

\section{Ionized jets and winds}

Hydrogen recombination lines such as $\operatorname{Br} \gamma$ are useful diagnostic tracers of the mass accretion and the stellar and disk winds. The $\operatorname{Br} \gamma$ lines in our targets show emission features with $|v| \sim 100-200 \mathrm{kms}^{-1}$ and a high-velocity P-Cygni profile $\left(-800 \mathrm{kms}^{-1}\right.$ to $-300 \mathrm{kms}^{-1}$ ) in V645 Cyg. Position discrepancies between the blueshifted and redshifted components are detected in IRAS 18151-1208 and S106 IRS4. Our interpretations on these results are as follows. For IRAS 18151-1208, these components probe the bipolar jet. The orientation and the velocity are consistent with those detected in the shock excited $\mathrm{H}_{2}$ jet. For S106 IRS4, the orientation is in the disk plane and consistent with the disk wind seen in radio.

\section{Rotating CO torus}

In IRAS 18151-1208 and AFGL 2136, series of $R$ and $P$ branch of the CO $\nu=2-0$ absorption lines are detected around $2.33 \mu \mathrm{m}$. The images of these lines show position discrepancies between the blueshifted and redshifted components. This is likely to be due to the Keplerian rotating motion of the neutral gas disks or tori. We calculate the velocity centroid integrating through the direction perpendicular to the rotating axis. The velocity centroid images show $\pm 15( \pm 10) \mathrm{kms}^{-1}$ for IRAS $18151-1208$ and $\pm 10( \pm 7) \mathrm{kms}^{-1}$ for AFGL 2136. We also analyze the position-velocity (PV) relationship and find a skewness in the PV map, which is known to be the signature of Keplerian rotating motion.

In IRAS 18151-1208, the orientation of the rotating motion is perpendicular to the jet direction see in our $\operatorname{Br} \gamma$ data. The PV relationship can be explained with a model with a central mass of $30 M_{\odot}$. In AFGL 2136, the orientation of the rotating motion is somewhat uncorrelated to the $\mathrm{CO}$ outflow direction and the NIR polarization disk. Nevertheless, the central mass is estimated to be $20 M_{\odot}$. 